\title{
Professor Dr. Wiebecke neuer Präsident des Deutschen Verbandes Forstlicher Forschungsanstalten
}

\begin{abstract}
Dr. forest. Claus Wiebecke, o. Professor für Weltforstwirtschaft der Universität Hamburg und Direktor des Instituts für Weltforstwirtschaft der Bundesforschungsanstalt für Forst- und Holzwirtschaft in Reinbek, wurde zum Präsidenten des Deutschen Verbandes Forstlicher Forschungsanstalten gewählt und hat am 1. Januar 1972 die Geschäfte seines neuen Amtes übernommen. Er wird damit Nachfolger von o. Professor Dr. Dr. Dr. h. c. Kurt Mantel, Direktor des Instituts für Forst- und Holzwirtschaftspolitik der Forstwissenschaftlichen Fakultät der Albert-Ludwigs-Universität in Freiburg/Breisgau, der nach 20 Jahren Tärigkeit als Präsident auf eine Wiederwahl verzichtet hat.
\end{abstract}

\section{BUCHBESPRECHUNGEN}

A Manual of the Dipterocarp Trees of Brunei State and of Sarawak-Supplement. (Leitfaden der Dipterocarpaceen-Bäume in Brunei und Sarawak - Ergänzungsband.) By P. S. Ashton, Borneo Literature Bureau, 1968. 129 S., 23 Bildtafeln, 15 Abb.

Dieses Buch ergänzt ein 1964 erschienenes Werk desselben Autors, das nur die Dipterocarpaceen Bruneis behandelte. Es beschreibr und illustriert etwa 200 baumförmige Arten der Gattungen Dipterocarpus, Cotylelobium, Vatica, Parashorea, Hopea und Shorea und behandelt damit einige der wichtigsten Nutzhölzer im tropischen Asien.

Dem eigentlichen Text werden Gattungs- und Artschlüssel vorangestellt; das Hauptgewicht liegt jedoch auf den Artbeschreibungen, die durch Strichzeichnungen von Blättern und Früchten ergänzt werden und die neben einer eingehenden Herbarbeschreibung Ausführungen über die Verbreitung, über die wirtschaftliche Bedeutung und über eine Schnelldiagnose enthalten. Die wichtigsten Arten werden darüber hinaus in ganzseitigen Schwarz-Weiß-Photos abgebildet.

P. Sснӥтr

Pflanzenphysiologie. Molekulare und biochemisch-physiologische Grundlagen von Stoffwechsel und Entwicklung. Von Prof. Dr. Dieter Hess. Stuttgart: Eugen Ulmer 1970. 367 S., 248 meist 2farb. Abb., flex. Kunststoffeinband 19,80 DM.

Das Taschenbuch erobert sich mehr und mehr Bereiche, die früher ausschließlich dem biederen Lehrbuch vorbehalten waren. Der guten Gründe dafür sind gar viele, und so verwundert es nicht, wenn nun ein solches auch über "Pflanzenphysiologie" vorliegt. Dies ist um so mehr zu begrüßen, als der Verfasser in überzeugender Weise die molekularbiologische und biochemische Sicht besonders herausstellt. Die Darstellung der oft äußerst verwidkelten Zusammenhänge ist klar, anschaulich, übersichtlich und knapp. Wer zu lesen beginnt, legt das Taschenbuch nur ungern zur Seite, weil es stets zu eigenem Mitdenken anregt und selbst dem Erfahrenen nicht langweilig wird. Besonders hervorzuheben ist das Bemühen, ein lebendiges Bild 